\title{
Harnessing Synthesized Abstraction Images to Improve Facial Attribute Recognition
}

\author{
Keke He $^{1 *}$, Yanwei Fu ${ }^{2 *}$, Wuhao Zhang ${ }^{4}$, Chengjie Wang ${ }^{3}$, \\ Yu-Gang Jiang ${ }^{1 \dagger}$, Feiyue Huang ${ }^{3}$, Xiangyang Xue ${ }^{1}$ \\ ${ }^{1}$ School of Computer Science, Shanghai Key Lab of Intelligent Information Processing, Fudan University \\ ${ }^{2}$ School of Data Science, Fudan University \\ ${ }^{3}$ Tencent Youtu Lab \\ ${ }^{4}$ Shanghai Jiao Tong University \\ \{kkhe15, yanweifu\}@fudan.edu.cn,vv_xiaod@sjtu.edu.cn,jasoncjwang@tencent.com, \\ ygj@fudan.edu.cn,garyhuang@tencent.com,xyxue@fudan.edu.cn
}

\begin{abstract}
Facial attribute recognition is an important and yet challenging research topic. Different from most previous approaches which predict attributes only based on the whole images, this paper leverages facial parts locations for better attribute prediction. A facial abstraction image which contains both local facial parts and facial texture information is introduced. This abstraction image is generated by a Generative Adversarial Network (GAN). Then we build a dual-path facial attribute recognition network to utilize features from the original face images and facial abstraction images. Empirically, the features of facial abstraction images are complementary to features of original face images. With the facial parts localized by the abstraction images, our method improves facial attributes recognition, especially the attributes located on small face regions. Extensive evaluations conducted on CelebA and LFWA benchmark datasets show that state-ofthe-art performance is achieved.
\end{abstract}

\section{Introduction}

Facial attribute recognition has received extensive research attention over the past decades. Facial attributes are used to describe the person characteristics of a face image. Learning to predict facial attributes can not only be used as the intermediate representations for other learning tasks such as face recognition [Wang et al., 2017b; Hu et al., 2017], but also directly useful for real-world applications such as face retrieval [Siddiquie et al., 2011], and intelligent retail. For example, analyzing facial attributes can automatically detect the age and gender of customers in the shopping malls and thus helps these commercial agents to accumulate and understand the Big Data of customer styles.

${ }^{*}$ This work was done when Keke He was an intern at Tencent Youtu Lab. The first two authors contributed equally to this paper.

${ }^{\dagger}$ Yu-Gang Jiang is the corresponding author.
Learning a robust model for facial attribute recognition is very challenging primarily due to the difficulties of parsing input face images. Specifically, the input face images may contain very noisy and dynamic background, e.g., the scene of a shopping mall. This background information may negatively affect the recognition process of facial attributes. Furthermore, most types of facial attributes (e.g., eyeglasses, or arched eyebrows) can be localized to some particular regions of faces. For example, the "wearing hat" attribute is mostly corresponding to the hair part of human faces without needing the information from other parts of the image, say, the mouth. Isolating the local regions to learn each type of attribute can help facial attribute recognition.

To directly parse the local parts of faces, previous works either use the landmarks to crop face region by bounding box [Kumar et al., 2009], or directly segment the face images into facial parts [Kalayeh et al., 2017]. The former methods may include the undesirable parts. For instance, if using the bounding box to crop the hair part, it may crop the whole face region if the person has long hair. The latter one may result in losing the details of texture information. This detailed information is nevertheless very critical for facial attribute recognition. In contrast, this paper "isolates" the important factor to predict the facial attributes with the facial abstraction task. We aim at generating abstracted facial regions from original face images that is possible to remove the useless background but still contains the facial part locations information.

The facial abstraction task is inspired by the task of facial segmentation which parses the face images into meaningful facial parts. The key difference is that our facial abstraction task will require the parsing algorithm to save as much texture information from the original images as possible. Essentially, facial abstraction process can be implemented by the recent Generative Adversarial Network (GAN) model [Goodfellow et al., 2014]. After obtaining the synthesized facial abstraction image, the original image and abstracted image are fed into a dual-path network which contains original image subnet and abstraction image subnet. To further leverage the information from the abstraction sub- 


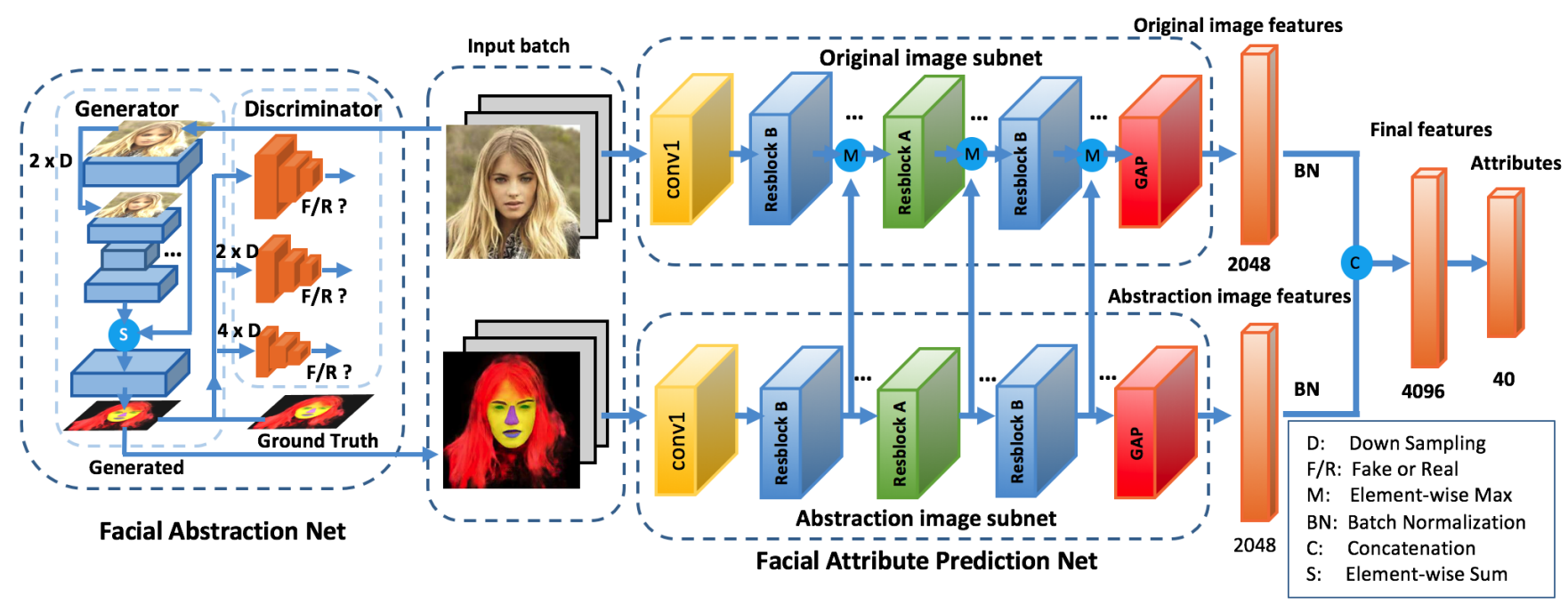

Figure 1: Overview of the proposed architecture. The facial abstraction net is based on pix2pixHD.

net, the feature maps of the abstraction subnet are passed to original image subnet. Finally, these two features are concatenated for final attribute recognition. Our attribute recognition network is trained in an end-to-end manner. We evaluate our proposed framework on benchmarks including CelebA [Liu et al., 2015b], LFWA [Huang et al., 2007; Liu et al., 2015b] face attribute datasets and the experiment results significantly outperform the state-of-the-art alternatives.

In summary, our main contribution is to propose a systematic way of harnessing synthesized abstraction images to help improve facial attribute recognition. In particular, (1) To the best of our knowledge, we for the first time utilize the GAN model to generate the facial abstraction images which contain the part locations and textual information. (2) We are the first to propose a dual-path network to combine the synthesized abstraction images and original images to help attribute recognition. (3) We show that attribute recognition can be improved with the help of abstraction images. We evaluate the framework on two benchmark datasets, and the experimental results validate the effectiveness of our method.

\section{Related Work}

Facial Attribute Recognition. In term of distinctive learning paradigm, the facial attribute recognition can be divided into two categories: part-based and holistic approaches. For the part-based method, it contains an attribute-related part detector and then extracts features on the localized facial parts. [Kumar et al., 2009] employed hand-crafted features to parse pre-defined facial parts to facilitate training SVM for facial attribute recognition. [Zhang et al., 2014] employed poselets [Bourdev et al., 2011] to detect body parts to extract Convolutional Neural Network (CNN) features of the localized parts.

On the other hand, various deep multi-task architectures [Liu et al., 2015b; Rudd et al., 2016; Lu et al., 2017; Han et al., 2017] are holistically learned for facial attribute recognition. Comparing with all these previous methods, the GAN model is learned in our framework to parse facial parts to better help attribute prediction. [Ding et al., 2017] designed a weakly-supervised face region aware network to automatically detect face regions, while ours learns a GAN to obtain parts locations.

[Kalayeh et al., 2017] employed the semantic segmentation to improve facial attribute prediction; in contrast, we utilize synthesized abstraction images. Specifically, (1) Different frameworks to generate segmentation/abstraction: [Kalayeh et al., 2017] adopted an encoder-decoder in generating the segmentation, rather than the synthesized abstraction images produced by GAN in our framework. (2) Different ways of using segmentation/abstraction for prediction. The segmentation images are used in [Kalayeh et al., 2017] as masks to pool/gate the activations (features) for prediction. In contrast, our synthesized abstraction images are directly used to train a network for attribute prediction. Critically, the network trained by synthesized images is able to achieve relative competitive results compared with the other baselines as shown in Tab. 1, Tab. 3, Tab. 4 and Tab. 5.

Face Segmentation and Face Inpainting. Face segmentation is also called as face parsing. It gives a semantic class label to every pixel in a face image, results in segmenting the input face image into semantic regions, e.g, hair, eyes and nose for further analysis. Researchers have developed several face segmentation methods based on Conditional Random Field (CRF), exemplar [Smith et al., 2013] and deep neural network [Liu et al., 2015a]. For the exemplar-based methods, [Smith et al., 2013] proposed a method based on transferring labeling masks from registered exemplars images to the test image in a pixel-wise manner. For the deep neural network based methods, [Luo et al., 2012] developed a deep parsing framework based on deep hierarchical features and separately trained models. [Liu et al., 2015a] proposed a multi-objective deep network that can jointly learn pixelwise likelihoods and pairwise label dependencies. Similarly, face inpainting refers to the technique of modifying a face 
image with partial occlusions due to sunglasses and hand in a seamless manner. An early attempt to inpainting is by [Mo et al., ], which reconstructed the occluded region of a face by a linear combination of several face images. Recently, [Jampour et al., 2017] introduced a data-driven approach which made use of inferred high-level facial attributes, such as gender, ethnicity, and expression. There are some methods which use generative model to inpainting [Pathak et al., 2016; Yeh et al., 2017]. [Yeh et al., 2017] proposed a method that learned to generate the missing content by searching for the closest encoding of the corrupted image in the latent image manifold. Different from face segmentation and face inpainting tasks, our facial abstraction task not only generates the facial parts but also contains an amount of textual information. The generated results are basically learned and abstracted from a large amount of training data. Thus the abstracted image results are not only based on the input image but also get affected by those images that are mostly similar to the input image.

\section{Methodology}

We propose a dual-path deep convolutional neural network for facial attribute recognition. The framework is illustrated in Fig. 1. It is composed of a facial abstraction network and a facial attribute prediction network. The facial attribute prediction network is composed of two subnets. The features of two subnets are concatenated after batch normalization [Ioffe and Szegedy, 2015]. The concatenated features are used for the final attribute recognition by a sigmoid cross entropy loss layer. Each component will be described next, including the face attribute recognition problem in Sec. 3.1 and the structure of base attribute recognition network in Sec. 3.2. Then we will introduce the way to generate abstraction images in Sec. 3.3. Finally, the training process will be discussed in Sec. 3.4.

\subsection{Problem Setup}

We aim to learn the attribute classifiers that can predict the existence of attributes of face images. Assume we have the training dataset $\mathcal{D}_{s}=\{\mathbf{I}, \mathbf{a}, \mathbf{L}\}$ with $N$ training images and $M$ attributes. I denotes the training instances, $\mathbf{a}$ is the attribute names and $\mathbf{L}$ denotes the labels. If the $i$-th image $\mathbf{I}_{i},(i=1, \cdots, N)$ is annotated to have the $j$-th attribute $\mathbf{a}_{j}(j=1, \cdots, M)$, we denote $\mathbf{L}_{i j}=1$; otherwise, $\mathbf{L}_{i j}=0$. Given a unseen test image $\mathbf{I}^{\star}$, the goal is then to learn a mapping function $\mathbf{a}^{\star}=\Psi\left(\mathbf{I}^{\star}\right)$ using all available training information and predict the attribute vector $\mathbf{a}^{\star}$. As each image can be labelled with multiple attributes, the predicting functions can be written as $\Psi=\left[\psi_{j}\right]_{j=1, \cdots, M}$, and $\psi_{j}\left(\mathbf{I}^{\star}\right) \in\{+1,0\}$.

\subsection{Basic Attribute Prediction Network}

Our basic attribute prediction network is illustrated in Fig. 2. It includes the convolutional layers, pooling layers, fully connected layers and residual block layers [He et al., 2015].

Convolutional Layers. This type of layer pre-processes the input image for the following steps. In particular, the first convolutional layer is set as $7 \times 7 \mathrm{kernel}$ size in order to guarantee a large receptive field. For all the other convolutional



Figure 2: The structure of our basic attribute prediction network. It is based on ResNet50. Note that: GAP represents the global average pooling layer.

layers, the kernel size is $3 \times 3$. Except for the first convolutional layer, all the other convolutional layers are used to construct two types of residual blocks - Resblock A and Resblock B.

Residual Block Layers. For all the residual blocks, it has 3 convolutional filters as the main road. (1) In ResBlock A, there is one convolutional filter on the side road. (2) In ResBlock B, there is a bypass directly to the output. Finally, these two roads are connected by an element-wise sum operation. After the final residual block, Global Average Pooling (GAP) layer is applied to produce a 2048-D vector representation.

Fully Connected Layers. This layer converts 2048-D features to $\mathbf{M}$ attribute values, where $\mathbf{M}$ is the number of attributes. This basic structure is used to construct two subnets in our dual-path attribute prediction model. In the previous methods, the Euclidean loss is used as the loss function [Rudd et al., 2016]. In contrast, we train the network using sigmoid cross entropy loss, which is shown better at predicting the facial attributes in the experiments.

\subsection{Facial Abstraction Network}

The facial abstraction network aims at synthesizing an abstraction of the image from the original image. The recent GAN-based method is applied for such purpose. Essentially, GAN has two components: the generator and the discriminator. The generator aims at learning to synthesize images that are indistinguishable from the natural images, while the discriminator is optimized to differentiate the synthesized images from the real natural images. In particular, we utilize the pix2pixHD [Wang et al., 2017a] to learn to generate the facial abstraction image. It has two components: a coarse-to-fine generator $G$ and a multi-scale discriminator $D$.

The pix2pixHD tries to produce a realistic natural image by a given segmentation image. In contrast, as shown in Fig. 1, our method takes the natural images as the input and generates the abstracted face images. Our training data is a set of pairs of images $\left(r_{i}, a_{i}\right)$, where $r_{i}$ is the real images and $a_{i}$ is the corresponding abstraction images. Our GAN aims to model the conditional distribution of abstraction images 




Figure 3: Comparing the face segmentation and abstraction results on Helen testing set. The first row is the original images. The second row is the segmentation result generated by Deeplabv2; images are colored by the 11 ground truth labels. The third row is our facial abstraction result.

given the real images by the following objective function,

$$
\begin{array}{r}
\operatorname{minmax}_{G} \mathcal{L}_{G A N(G, D)}=\mathbb{E}_{r, a \sim p_{\text {data }}(r, a)}[\log D(r, a)] \\
+\mathbb{E}_{r \sim p_{\text {data }}(r)}[\log (1-D(r, G(r)))],
\end{array}
$$

In particular, the pix2pixHD used 3 discriminators $\left(D_{1}, D_{2}\right.$ and $D_{3}$ ) corresponding to three scales of images. The downsampling operators are conducted on the real and synthesized images by a factor of 2 and 4 respectively, in order to get the images used for $D_{2}$ and $D_{3}$ respectively. Thus we can formulate learning GAN as a multi-task learning problem as,

$$
\min _{G} \max _{D_{1}, D_{2}, D_{3}} \sum_{k=1}^{3} \mathcal{L}_{G A N\left(G, D_{k}\right)}
$$

Our facial abstraction images are also compared against the results of face segmentation produced by Deeplabv2 [Chen $e t$ al., 2016]. The visualization results are shown in Fig. 3. The first, second and third rows are the images of original, face segmentation and facial abstraction respectively. Apparently, the facial abstraction images have saved more texture information than segmentation images. The details of training procedure will be described in 4.1 .

\subsection{Training Process}

In this section, we will describe the training process which leverages information from the facial abstraction images. The original face images and abstraction images are paired as the input to the network, these two kinds of images go to the two subnets: original image subnet and abstraction image subnet respectively. As these two images have a different visual appearance, to fully explore the information in the two input images, the weights of two subnets are unshared. Global average pooling is applied after the last residual block layer and then the features from two subnets are obtained. Additional batch normalization layer is added to normalize two types of features. The normalized features are concatenated to generate the final features to predict attributes. In order to better leverage the abstraction image information, the connections from the abstraction subnet to the original subnet are added. Specially, after each residual block, the output feature maps of the abstraction image are passed to the original subnet. These two outputs are fused by an element-wise max operation.

\section{Experiments}

\subsection{Datasets and Experimental Settings}

We conduct experiments on two most widely used datasets. (1) CelebA contains 202,599 images of approximately 10k identities [Liu et al., 2015b]. Each image is annotated with 40 binary attributes. For a fair comparison with the other methods, we follow the standard split here: the first 162,770 images are used for training, 19, 867 images for validation and remaining 19, 962 for testing. CelebA provides the precropped face images and we use cropped images to train and test attribute models same as the other methods [Rudd et al., 2016]. (2) LFWA [Liu et al., 2015b] is constructed based on face recognition dataset LFW [Huang et al., 2007]. LFWA has a total of 13,232 images of 5,749 identities with predefined train and test splits which divide the entire dataset into two approximately equal partitions. We follow the partition of data to train and test our model. In LFWA, each image has 40 binary facial attributes, the same as CelebA.

Evaluation Metrics. The facial attribution recognition can be taken as the problem of classification tasks. To evaluate the performance, (1) mean accuracy ( $a c c$ ) over all attributes is computed. This metric has also been used in previous work [Liu et al., 2015b]. (2) Further, we find the positive and negatives instances per attribute are extremely imbalanced in the CelebA dataset. For example, for the "Bald" attribute, we can get a high accuracy of $97.88 \%$ if predicting all the test images have no bald. To appropriately evaluate the quality of different methods, following the evaluation metrics used in pedestrian attribute recognition problem [Li et al., 2016], we add four more evaluation metrics, a label-based metric mean balanced-accuracy, short in bal-acc, and three instance-based metrics, i.e. precision (prec), recall (rec) and F1-score (FI). Formally, the acc and bal-acc can be calculated as,

$$
\begin{gathered}
a c c=\frac{1}{M} \sum_{i=1}^{M}\left(T_{i} / N\right) \\
b a l-a c c=\frac{1}{2 M} \sum_{i=1}^{M}\left(T P_{i} / P_{i}+T N_{i} / N_{i}\right)
\end{gathered}
$$

where $M$ is the total number of attributes; $N$ and $T_{i}$ are the numbers of examples and correctly predicted examples; $P_{i}$ and $T P_{i}$ are the numbers of positive examples and correctly predicted positive examples; $N_{i}$ and $T N_{i}$ are the numbers of negative examples and correctly predicted negative examples. Parameter Settings. We use the open source deep learning framework Caffe [Jia et al., 2014] to train our network. For all the experiments, we only use a single end-to-end model for testing. We use the stochastic gradient descent algorithm to train our models. (1) On CelebA dataset, the weights of convolutional layers are initialized by the ResNet50 [He et 
Proceedings of the Twenty-Seventh International Joint Conference on Artificial Intelligence (IJCAI-18)

\begin{tabular}{|c|c|}
\hline Methods & Accuracy $(\%)$ \\
\hline [Kumar et al., 2008] FaceTracer & 81.12 \\
\hline [Zhang et al., 2014] PANDA-w & 79.00 \\
\hline [Zhang et al., 2014] PANDA-1 & 85.00 \\
\hline [Liu et al., 2015b] LNets+ANet & 87.30 \\
\hline [Ehrlich et al., 2016] MT-RBM-PCA & 87.00 \\
\hline [Zhong et al., 2016] Off-the-Shelf CNN & 86.60 \\
\hline [Wang et al., 2016] Walk-and-Learn & 88.00 \\
\hline [Rudd et al., 2016] Rudd et al. Separate & 90.22 \\
\hline [Rudd et al., 2016] Rudd et al. Moon & 90.94 \\
\hline [Lu et al., 2017] SOMP-branch-32 & 90.74 \\
\hline [Hand and Chellappa, 2017] MCNN-AUX & 91.26 \\
\hline [Ding et al., 2017] $\mathrm{PaW}$ & 91.23 \\
\hline [Kalayeh et al., 2017] Avg. Pooling & 90.86 \\
\hline [Kalayeh et al., 2017] SSG & 91.62 \\
\hline [Kalayeh et al., 2017] SSP & 91.67 \\
\hline [Kalayeh et al., 2017] SSP+SSG & 91.80 \\
\hline Original & 91.50 \\
\hline Abstraction & 90.36 \\
\hline Ours & 91.81 \\
\hline
\end{tabular}

Table 1: Comparison of mean accuracy on CelebA with state-of-theart methods.

al., 2015] network that is pre-trained on ImageNet dataset. The base learning rate is set as 0.001 and gradually decreased by $1 / 10$ at $20 k, 45 k$ iterations. The input image is resized to $224 \times 224$. (2) On LFWA dataset, due to the relatively small number of training samples $(6 k)$, we adopt a smaller network structure ResNet18 [He et al., 2015] to avoid overfitting. The base learning rate is still set as 0.001 and gradually decreased by $1 / 10$ at $1 k, 2 k$ iterations.

Running Costs. Our model trained on CelebA dataset gets converged with $46 k$ iterations and it takes 10 hours with one NVIDIA Tesla M40 GPU. Our model trained on LFWA gets converged with $2.5 k$ iterations and it takes half an hour. For training all the model, the batch size is 20 , and it takes around 22 GB GPU memory.

Facial Abstraction Networks. This network is trained by the Helen dataset, which is a widely used dataset for face parsing. [Le et al., 2012; Smith et al., 2013]. In this dataset, each image is annotated with 11 segment classes. These labels are as follows: background, face skin (excluding ears and neck), left eyebrow, right eyebrow, left eye, right eye, nose, upper lip, inner mouth, lower lip, and hair. It is composed of total 2,330 images and divided into 2,000 training images, 230 validation images, and 100 testing images. We train face abstraction model on the training images. To generate the ground truth abstraction images, we use the codes of [Liu et $a l ., 2015 \mathrm{a}]$ which saves the textual information. To train the facial abstraction model, we use the codes of [Wang et al., 2017a]. As our GAN learns the distribution of training data (including textual information), we can generate images with textual information. Our input image and the label image are resized to $256 \times 256$. We use Adam with the learning rate of 0.0002 to optimize our abstraction network. The batch size is 1. We train the network with 200 epochs. It takes 37 hours

\begin{tabular}{c|c|c}
\hline Attributes & Original Images(\%) & Our Model (\%) \\
\hline \hline ArchedEyebrows & 84.28 & $\mathbf{8 5 . 1 5}$ \\
\hline BagsUnderEyes & 85.27 & $\mathbf{8 5 . 7 7}$ \\
\hline BushyEyebrows & 92.77 & $\mathbf{9 3 . 0 7}$ \\
\hline Eyeglasses & 99.68 & $\mathbf{9 9 . 7 2}$ \\
\hline NarrowEyes & $\mathbf{8 7 . 8 5}$ & 87.81 \\
\hline
\end{tabular}

Table 2: Comparison of eye/eyebrow related attributes on CelebA with baseline model.

\begin{tabular}{c|c}
\hline Methods & Accuracy $(\%)$ \\
\hline \hline [Kumar et al., 2008] FaceTracer & 73.93 \\
\hline [Zhang et al., 2014] PANDA-w & 79.00 \\
\hline [Zhang et al., 2014] PANDA-1 & 81.00 \\
\hline [Liu et al., 2015b] LNets+ANet(w/o) & 79.00 \\
\hline [Liu et al., 2015b] LNets+ANet & 84.00 \\
\hline [Kalayeh et al., 2017] Avg. Pooling & 85.27 \\
\hline [Kalayeh et al., 2017] SSG & 86.13 \\
\hline [Kalayeh et al., 2017] SSP & 86.80 \\
\hline [Kalayeh et al., 2017] SSG+SSP & $\mathbf{8 7 . 1 3}$ \\
\hline \hline ResNet18+ SVM & 82.35 \\
\hline ResNet50 + SVM & 83.09 \\
\hline Original & 84.79 \\
\hline Abstraction & 83.64 \\
\hline Ours & 85.28 \\
\hline
\end{tabular}

Table 3: Comparison of mean accuracy on LFWA datasets with state-of-the-art methods.

with one NVIDIA Tesla M40 GPU and needs around 13 GB GPU memory. We then apply the abstraction network to the face attribute recognition datasets. Even very few numbers of Helen training data used in our training process, the abstraction model is able to color various facial regions successfully in unseen images. Later, we evaluate our proposed attribute prediction model where these abstraction cues are utilized to improve facial attribute recognition.

\subsection{Competitors}

We compare our results against state-of-the-art methods and baselines. Particularly, (1) FaceTracer [Kumar et al., 2008] extracts the HOG and color histograms in manually defined facial parts and then trains SVM for each attribute recognition. (2) PANDA [Zhang et al., 2014] uses poselets [Bourdev et al., 2011] to detect parts and then extracts CNN features from the localized parts. (3) LNets+ANet [Liu et al., 2015b] employs two deep CNNs to localize face and one deep CNN network to learn facial feature. (4) Off-the-Shelf CNN [Zhong et al., 2016] extracts features from the off-the-shelf face recognition model. (5) Walk and Learn [Wang et al., 2016] exploits videos and contextual data to learn representations for facial attributes. (6) Moon [Rudd et al., 2016] learns a mixed objective optimization network for learning each attribute and utilizes distribution of attribute labels. (7) SOMP [Lu et al., 2017] learns a deep multi-task learning framework which can dynamically group similar tasks together. (8) MCNN-AUX [Hand and Chellappa, 2017] takes the attribute 


\begin{tabular}{c|c|c|c|c|c}
\hline Methods & Acc. & Bal-acc. & Prec. & Rec. & F1 \\
\hline \hline Original & 91.50 & 81.57 & $\mathbf{8 4 . 6 7}$ & 76.20 & 80.21 \\
\hline Abstraction & 90.36 & 78.64 & 82.11 & 73.66 & 77.66 \\
\hline Our Model & $\mathbf{9 1 . 8 1}$ & $\mathbf{8 3 . 1 3}$ & 84.22 & $\mathbf{7 8 . 2 1}$ & $\mathbf{8 1 . 1 0}$ \\
\hline
\end{tabular}

Table 4: Comparison with the baseline models on CelebA.

\begin{tabular}{c|c|c|c|c|c}
\hline Methods & Acc. & Bal-acc. & Prec. & Rec. & F1 \\
\hline \hline Original & 84.79 & 76.48 & 80.90 & 74.19 & 77.40 \\
\hline Abstraction & 83.64 & 75.05 & 79.09 & 72.52 & 75.67 \\
\hline Our Model & $\mathbf{8 5 . 2 8}$ & $\mathbf{7 7 . 5 0}$ & $\mathbf{8 2 . 0 1}$ & $\mathbf{7 4 . 2 2}$ & $\mathbf{7 7 . 9 2}$ \\
\hline
\end{tabular}

Table 5: Comparison with the baseline models on LFWA.

relationships into consideration for improving classification accuracy. (9) PaW [Ding et al., 2017] combines multiple part-based networks and a whole-image-based network for final attribute classification. (10) [Kalayeh et al., 2017] learns an encoder-decoder to produce the segmentation images, and then leverages the segmentations as masks to pool/gate the activations for attribute prediction. Here we compare three different variants of [Kalayeh et al., 2017] — Average Pooling, SSG, SSP. These three variants configure three different ways of utilizing the segmented images to pool/gate the feature maps and thus help facial attribute recognition. (11) Original is one variant of our model. Original images are used as input to train the model. (12) Abstraction is another variant of our model. It uses the abstraction image as the input. (13) ResNet18 + SVM is one baseline model. It extracts the features from a whole face image by a ResNet 18 model which pre-trained on ImageNet2012, and then trains one SVM classifier for each attribute. (14) ResNet50 + SVM is another baseline model. Features from ResNet50 model pre-trained on ImageNet2012 are used to train separate SVM for each attribute.

\subsection{Results on CelebA Dataset}

We evaluate the facial attribute recognition task with the standard settings of CelebA dataset. The results are listed in Tab. 1. We highlight the following observations.

(1) State-of-the-art results. The results of our model beat all the state-of-the-art methods. Comparing with all the other methods, we highlight that our method achieves the best performance with the mean accuracy of $91.81 \%$ over 40 facial attributes. The results show $5.21 \%, 3.81 \%$ and $0.89 \%$ improvement over Off-the-Shelf [Zhong et al., 2016], Walkand-Learn [Wang et al., 2016], Moon [Rudd et al., 2016] respectively. In particular, comparing with the current stateof-the-art method LNets+ANet [Liu et al., 2015b] which has a classification error of $12.70 \%$, our method with an error of $8.19 \%$, reducing the classification error by $35.5 \%$. This improved performance validates the effectiveness of our framework. It is important to note that, [Wang et al., 2016] used 5 million auxiliary image pairs to pre-train their model, and [Lu et al., 2017] employed the face recognition model as the pre-train model.

(2) Effectiveness of facial abstraction subnet. We compare the other variants of our model and show the efficacy of ab- straction subnet. Specifically, we compare several baseline models: Original and Abstraction, we find that even training with the abstraction images, our abstraction baseline model can get a mean accuracy of $90.36 \%$, which can beat the most of the state-of-the-art methods. This validates our abstraction images can well represent the original images, preserving the detailed facial information. Besides, our dual-path model can obtain a mean accuracy of $91.81 \%$, and it shows $1.45 \%$, $0.31 \%$ improvement over the abstraction and original model baselines individually. This is due to the fact that the features of original image and abstraction image are complementary to each other. And more critically, our dual-path network can efficiently combine them to produce very competitive results. (3) Finally, we compare our results with [Kalayeh et al., 2017]. In particular, (1) We highlight that this is the first work of utilizing synthesized images to help facial attribute prediction. The synthesized images are capable of training a network in attribute prediction. We further harness these synthesized images to improve the performance. (2) Both methods are very good, and yet we are using different strategies in the way of generating segmentation/abstraction images and using segmentation/abstraction for prediction. Compared with the mean accuracy, our results are very marginally better than [Kalayeh et al., 2017] on CelebA dataset, and slightly worse on LFWA dataset. Note that the CelebA dataset which has of $162 k$ and $20 k$ images for training and testing individually is much larger than the LFWA dataset of $6 \mathrm{k}$ training and $7 \mathrm{k}$ testing images. This shows that our methods are comparable to the state-of-the-art methods in [Kalayeh et al., 2017].

\subsection{Results on LFWA Dataset}

To further test the proposed method, we applied it to the LFWA face attribute dataset. We find that (1) Again the results of our model are better than or have comparable performance to the state-of-the-art methods. As we can see from Tab. 3, ours achieve the mean accuracy of $85.28 \%$ over 40 facial attributes. In particular, it shows $1.38 \%$ improvement over the current state-of-the-art LNets+ANet [Liu et al., $2015 \mathrm{~b}$ ]. This validates the effectiveness of the proposed attribute classification network. (2) Furthermore, this experiment still validates the efficacy of parsing subnet. We list the result of the baseline models in Tab. 5. Our model can obtain the mean accuracy of $85.28 \%$, and it shows $1.59 \%$ and $0.49 \%$ improvement over two baseline models: abstraction images and original images respectively. This validates the efficacy of the abstraction image features. It is complementary to original images features. Meanwhile, the abstraction image can help to aware the locations of different facial components, thus improving the attribute recognition accuracy. Our method also shows $2.93 \%$ and $2.19 \%$ improvement over two SVM baseline models.

\subsection{More Evaluation Metrics}

We further compare our results with the baselines on more metrics. In particular, for the significant imbalance classification task, especially the facial attribute recognition, mean classification accuracy is not the best evaluation metric. Thus extensive study by using different evaluation metrics has been conducted and compared in Tab. 4 and Tab. 5. These metrics 


\begin{tabular}{c|c|c|c|c|c}
\hline Methods & Acc. & Bal-acc. & Prec. & Rec. & F1 \\
\hline \hline W./O. norm & 91.53 & 81.59 & $\mathbf{8 4 . 4 4}$ & 76.53 & 80.29 \\
\hline W. norm & $\mathbf{9 1 . 8 1}$ & $\mathbf{8 3 . 1 3}$ & 84.22 & $\mathbf{7 8 . 2 1}$ & $\mathbf{8 1 . 1 0}$ \\
\hline
\end{tabular}

Table 6: Comparison of with / without feature normalization on CelebA. W./O. norm and W. norm represent the methods without and with normalization.

\begin{tabular}{c|c|c|c|c|c}
\hline Methods & Acc. & Bal-acc. & Prec. & Rec. & F1 \\
\hline \hline Euc. Loss & $\mathbf{9 1 . 5 1}$ & 79.36 & $\mathbf{8 6 . 9 0}$ & 73.45 & 79.61 \\
\hline S.C.E. Loss & 91.50 & $\mathbf{8 1 . 5 7}$ & 84.67 & $\mathbf{7 6 . 2 0}$ & $\mathbf{8 0 . 2 1}$ \\
\hline
\end{tabular}

Table 7: Comparison of different loss on CelebA. Euc. Loss and S.C.E. Loss indicate the Euclidean loss and Sigmoid Cross Entropy Loss respectively.

definitions are the same as those in pedestrian attribute recognition [Deng et al., 2014], including a label-based metric mean balanced accuracy (bal-acc) and three instance-based metrics precision (prec), recall (rec) and F1-score (F1). These metrics can systematically evaluate the performance of our methods over baselines.

For example, on CelebA dataset, our dual-path model can achieve the 77.50 bal-acc, which outperforms the abstraction and original baselines by 2.45 and 1.02 respectively. Furthermore, our dual-path model hits the $81.10 F 1$, which improves over the abstraction model and original models by 3.44 and 0.89 individually. On LFWA dataset, we report our $F 1$ results of 77.92, which beats the two baselines again. Thus overall our results are still better than the baseline models.

\section{Ablation Study}

Analysis of attributes on small face regions. To further evaluate the abstraction subnet, we select the attributes which related to eye or eyebrows on CelebA dataset. In a face image, these two face components always occupy limited regions. We list the accuracy results on Tab. 2. Comparing our model with baseline original image model, our model has improvement on all the attributes except the NarrowEyes attribute. This may reveal that with the help of abstraction images, our model can aware the small but important parts of facial images, thus improving the accuracy of these attributes. The choice of the loss function. We evaluate the loss function for binary attribute prediction network. [Rudd et al., 2016] uses the Euclidean loss to regress attribute labels, [Kalayeh et al., 2017] uses the sigmoid cross entropy loss to classify attributes. To evaluate which loss is better, we apply different loss on the CelebA dataset, the results are listed in Tab. 7. If compared with the mean accuracy metric, these two losses can achieve comparable results with $91.51 \%$ and $91.50 \%$ respectively. We further evaluate this two loss functions on mean balanced-accuracy, precision, recall and F1 metrics. Sigmoid cross entropy loss has 2.21 improvement on mean balanced-accuracy and 0.60 improvement on F1. Euclidean loss can only beat cross entropy loss on the precision metric. This reveals sigmoid cross entropy loss is better for binary attribute classification. Thus, we adopt sigmoid cross entropy loss to train all attribute models.
The importance of feature normalization. This study evaluates the importance of feature normalization. In our model, after the last pooling layer, the features of the face image and abstraction image are obtained. Before the feature concatenation, we compare our framework with feature normalization and without feature normalization. To perform feature normalization, we add additional batch normalization layer after the last pooling layer. The results are listed in Tab. 6. As we can see from the table, with feature normalization method can achieve $0.28 \%$ mean accuracy and $1.54 \%$ mean balanced-accuracy improvement over without feature normalization method. This reveals feature normalization is important before concatenation.

\section{Conclusion}

In this paper, we propose a novel dual-path convolutional neural network to learn facial attributes. Different from most previous approaches which predict attributes only based on the whole images, our method utilizes synthesized facial abstraction images to help attribute recognition tasks. The proposed framework fuses the features from original images and facial abstraction images to learn all the attributes tasks. We demonstrate our approach on the CelebA, LFWA attribute datasets, showing substantial improvement over the state-ofthe-art methods.

\section{Acknowledgments}

The authors would like to thank anonymous reviewers for their helpful comments. The authors are also grateful for valuable suggestions from Ying Tai and Yanhao Ge. This work was supported in part by National Key R\&D Program of China (No.2017YFC0803700), NSFC under Grant (No.61572138 \& No.U1611461) and STCSM Project under Grant (No.16JC1420400 \& No.2017SHZDZX01).

\section{References}

[Bourdev et al., 2011] Lubomir Bourdev, Subhransu Maji, and Jitendra Malik. Describing people: A poselet-based approach to attribute classification. In ICCV. IEEE, 2011.

[Chen et al., 2016] Liang-Chieh Chen, George Papandreou, Iasonas Kokkinos, Kevin Murphy, and Alan L Yuille. Deeplab: Semantic image segmentation with deep convolutional nets, atrous convolution, and fully connected crfs. arXiv:1606.00915, 2016.

[Deng et al., 2014] Yubin Deng, Ping Luo, Chen Change Loy, and Xiaoou Tang. Pedestrian attribute recognition at far distance. In ACM MM, 2014.

[Ding et al., 2017] Hui Ding, Hao Zhou, Shaohua Kevin Zhou, and Rama Chellappa. A deep cascade network for unaligned face attribute classification. In AAAI, 2017.

[Ehrlich et al., 2016] Max Ehrlich, Timothy J Shields, Timur Almaev, and Mohamed R Amer. Facial attributes classification using multi-task representation learning. In $C V P R$ Workshops, 2016.

[Goodfellow et al., 2014] Ian Goodfellow, Jean PougetAbadie, Mehdi Mirza, Bing Xu, David Warde-Farley, 
Sherjil Ozair, Aaron Courville, and Yoshua Bengio. Generative adversarial nets. In NIPS, 2014.

[Han et al., 2017] Hu Han, Anil K Jain, Shiguang Shan, and Xilin Chen. Heterogeneous face attribute estimation: A deep multi-task learning approach. TPAMI, 2017.

[Hand and Chellappa, 2017] Emily M Hand and Rama Chellappa. Attributes for improved attributes: A multi-task network utilizing implicit and explicit relationships for facial attribute classification. In $A A A I, 2017$.

[He et al., 2015] Kaiming He, Xiangyu Zhang, Shaoqing Ren, and Jian Sun. Deep residual learning for image recognition. arXiv:1512.03385, 2015.

[Hu et al., 2017] Guosheng Hu, Yang Hua, Yang Yuan, Zhihong Zhang, Zheng Lu, Sankha S Mukherjee, Timothy M Hospedales, Neil M Robertson, and Yongxin Yang. Attribute-enhanced face recognition with neural tensor fusion networks. In ICCV, 2017.

[Huang et al., 2007] Gary B Huang, Manu Ramesh, Tamara Berg, and Erik Learned-Miller. Labeled faces in the wild: A database for studying face recognition in unconstrained environments. Technical report, 2007.

[Ioffe and Szegedy, 2015] Sergey Ioffe and Christian Szegedy. Batch normalization: Accelerating deep network training by reducing internal covariate shift. In ICML, 2015.

[Jampour et al., 2017] Mahdi Jampour, Chen Li, Lap-Fai Yu, Kun Zhou, Stephen Lin, and Horst Bischof. Face inpainting based on high-level facial attributes. CVIU, 161:29 41, 2017.

[Jia et al., 2014] Y. Jia, E. Shelhamer, J. Donahue, S. Karayev, J. Long, R. Gir-shick, S. Guadarrama, and T. Darrell. Caffe: Convolutional architecture for fast feature embedding. arXiv, 2014.

[Kalayeh et al., 2017] Mahdi M Kalayeh, Boqing Gong, and Mubarak Shah. Improving facial attribute prediction using semantic segmentation. In CVPR, June 2017.

[Kumar et al., 2008] Neeraj Kumar, Peter Belhumeur, and Shree Nayar. Facetracer: A search engine for large collections of images with faces. In ECCV. Springer, 2008.

[Kumar et al., 2009] Neeraj Kumar, Alexander C Berg, Peter N Belhumeur, and Shree K Nayar. Attribute and simile classifiers for face verification. In ICCV, pages 365-372. IEEE, 2009.

[Le et al., 2012] Vuong Le, Jonathan Brandt, Zhe Lin, Lubomir Bourdev, and Thomas S Huang. Interactive facial feature localization. In ECCV. 2012.

[Li et al., 2016] Dangwei Li, Zhang Zhang, Xiaotang Chen, Haibin Ling, and Kaiqi Huang. A richly annotated dataset for pedestrian attribute recognition. arXiv:1603.07054, 2016.

[Liu et al., 2015a] Sifei Liu, Jimei Yang, Chang Huang, and Ming-Hsuan Yang. Multi-objective convolutional learning for face labeling. In $C V P R$, June 2015.
[Liu et al., 2015b] Ziwei Liu, Ping Luo, Xiaogang Wang, and Xiaoou Tang. Deep learning face attributes in the wild. In ICCV, 2015.

[Lu et al., 2017] Yongxi Lu, Abhishek Kumar, Shuangfei Zhai, Yu Cheng, Tara Javidi, and Rogerio Feris. Fullyadaptive feature sharing in multi-task networks with applications in person attribute classification. In CVPR, 2017.

[Luo et al., 2012] Ping Luo, Xiaogang Wang, and Xiaoou Tang. Hierarchical face parsing via deep learning. In CVPR. IEEE, 2012.

[Mo et al., ] Zhenyao Mo, John P Lewis, and Ulrich Neumann. Face inpainting with local linear representations.

[Pathak et al., 2016] Deepak Pathak, Philipp Krahenbuhl, Jeff Donahue, Trevor Darrell, and Alexei A Efros. Context encoders: Feature learning by inpainting. In $C V P R$, 2016.

[Rudd et al., 2016] Ethan M Rudd, Manuel Günther, and Terrance E Boult. Moon: A mixed objective optimization network for the recognition of facial attributes. In ECCV. Springer, 2016.

[Siddiquie et al., 2011] Behjat Siddiquie, Rogerio S Feris, and Larry S Davis. Image ranking and retrieval based on multi-attribute queries. In CVPR. IEEE, 2011.

[Smith et al., 2013] Brandon M Smith, Li Zhang, Jonathan Brandt, Zhe Lin, and Jianchao Yang. Exemplar-based face parsing. In CVPR, 2013.

[Wang et al., 2016] Jing Wang, Yu Cheng, and Rogerio Schmidt Feris. Walk and learn: Facial attribute representation learning from egocentric video and contextual data. In $C V P R, 2016$.

[Wang et al., 2017a] Ting-Chun Wang, Ming-Yu Liu, JunYan Zhu, Andrew Tao, Jan Kautz, and Bryan Catanzaro. High-resolution image synthesis and semantic manipulation with conditional gans. arXiv:1711.11585, 2017.

[Wang et al., 2017b] Zhanxiong Wang, Keke He, Yanwei Fu, Rui Feng, Yu-Gang Jiang, and Xiangyang Xue. Multitask deep neural network for joint face recognition and facial attribute prediction. In ICMR. ACM, 2017.

[Yeh et al., 2017] Raymond A Yeh, Chen Chen, Teck Yian Lim, Alexander G Schwing, Mark Hasegawa-Johnson, and Minh N Do. Semantic image inpainting with deep generative models. In CVPR, 2017.

[Zhang et al., 2014] Ning Zhang, Manohar Paluri, Marc'Aurelio Ranzato, Trevor Darrell, and Lubomir Bourdev. Panda: Pose aligned networks for deep attribute modeling. In CVPR, 2014.

[Zhong et al., 2016] Yang Zhong, Josephine Sullivan, and Haibo Li. Face attribute prediction using off-the-shelf $\mathrm{cnn}$ features. In ICB. IEEE, 2016. 